\title{
Oportunidades de aproximación al aula escolar de educación matemática como criterio de calidad de los programas de formación de profesores de educación básica
}

\author{
Eugenio del C. Chandía ${ }^{1 *}$, Gamal A. Cerda ${ }^{1}$, Carlos E. Pérez ${ }^{2}$ y Anahí A. Huencho ${ }^{3}$ \\ (1) Facultad de Educación, Universidad de Concepción, Concepción, Chile. \\ (Correo-e: echandia@udec.cl, gacerda@udec.cl) \\ (2) Instituto de Ciencias Sociales, Universidad de O'Higgins, Rancagua, Chile. (Correo-e: carlos.perez@uoh.cl) \\ (3) Facultad de Educación, Universidad Católica de Temuco, Temuco, Chile. (Correo-e: ahuencho@uct.cl)
}

* Autor a quien debe ser dirigida la correspondencia

Recibido Sep. 11, 2020; Aceptado Nov. 6, 2020; Versión final Dic. 20, 2020, Publicado Jun. 2021

\begin{abstract}
Resumen
El presente estudio tiene como objetivo examinar los syllabus de las asignaturas de matemática y didáctica de la matemática de 15 programas de formación docente de educación básica. Dado el cuestionamiento al sistema de aseguramiento de la educación en Chile, se condujo un análisis de correspondencia de los años de acreditación utilizando la unidad de comparación CARE (conocimiento matemático escolar, actividades, rol y espacio) de acercamiento al aula escolar. Los resultados muestran que independiente de los años de acreditación, solo una dimensión de la unidad de comparación se advierte en los syllabus de las asignaturas de todos los programas. Esto evidencia que tener más años de acreditación no asegura oportunidades anidadas. Se concluye que la dimensión de conocimiento matemático escolar de la unidad CARE de aproximación al aula escolar es la única que se observa de forma transversal en los programas de formación inicial de educación básica analizados.
\end{abstract}

Palabras clave: formación inicial docente; aula escolar; educación matemática; acreditación; aseguramiento de la calidad

\section{Approaching opportunities for mathematics classrooms as quality criteria for primary education teacher training programs}

\begin{abstract}
The main objective of the present study is to examine the syllabi of two primary school subjects, mathematics and didactic of mathematics, in 15 primary education teacher training programs. Given that the education assurance system in Chile is under close scrutinity, a correspondence analysis is performed to examine program accreditation years by using the comparison unit KARS (knowledge on school mathematics, activities, role, and space), as an approach to school classrooms. The results show that regardless of the years of accreditation, only one dimension of the comparison unit is observed in the course subjects of all the programs. This suggests that that having more years of accreditation does not ensure having nested opportunities. It is concluded that the KARS's dimension of knowledge on school mathematics is the only dimension observed across the teacher training programs examined.
\end{abstract}

Keywords: initial teacher training; school classroom; mathematics education; accreditation; quality assurance 


\section{INTRODUCCIÓN}

En la última década ha aumentado el interés por configurar procesos para asegurar la calidad de los sistemas educativos (Darling-Hammond, 2020; Han et al., 2020; Romanowski y Alkhateeb, 2020). Se han creado diversos medios para asegurar la calidad de los sistemas educativos, entre los que destacan aquellos afines a la Formación Inicial Docente (FID) como sistemas de regulación externa donde se establece la rendición de cuentas como medio para la asignación de responsabilidad (Darling-Hammond, 2020; Imig et al., 2016; Rowe y Skourdoumbis, 2017). Tanto es el impacto de estos sistemas que Ingvarson y Rowley (2017), al analizar los resultados de las evaluaciones TIMSS y PISA de los años 2015, encontraron que los países que tienen procesos de aseguramiento de la calidad más rigurosos de la FID obtuvieron resultados más exitosos en las pruebas. Del mismo modo, magros resultados en estas pruebas han sido atribuidos a la calidad de dichos programas de formación docente (Mayer et al., 2017).

Dentro de los mecanismos de aseguramiento de la calidad de los procesos de FID, existe una gran variabilidad respecto a cómo se forma el juicio evaluativo, atendiendo una diversidad de recursos, medios y procesos, entre los que se encuentran aquellos que centran su atención en el desarrollo de procesos de acreditación para verificar el cumplimiento de estándares de proceso y finalización para los estudiantes de pedagogía (Imig et al., 2016); aquellos que persiguen obtener indicadores que cuantifiquen el conocimiento disciplinar, pedagógico de la disciplina y pedagógico propiamente a través de evaluaciones de ingreso, proceso y egreso en la FID (Ingvarson y Rowley, 2017). También, algunos postulan medir la efectividad de la FID, a través de la observación de sus prácticas de instrucción y de los resultados de aprendizaje de los estudiantes (Ling, 2017); y otros postulan incorporar un control directo de los académicos formadores por medio de planes de gestión, cumplimiento de estándares y modelos curriculares (Lee y Day, 2016). También se cuentan aquellos que incorporan en su análisis la generación de evidencia mediante investigación y verificación de la reputación del programa de FID (Imig et al., 2016). Pero pese a esta variabilidad, un aspecto común de los mecanismos de aseguramiento es la observación y evaluación del tiempo clínico, su supervisión y aproximación a él (Klemenz, et al., 2019; Pushpanadham, 2020; Werler y Tahirsylaj, 2020)

La acreditación se comprende como un medio para el aseguramiento de la calidad de los sistemas educativos, de rendición de cuentas, auditoría y regulación, teniendo como principal propósito asegurar la calidad de los procesos formación inicial (Darling-Hammond, 2020; Ingvarson y Rowley, 2017; Romanowski y Alkhateeb, 2020; Rowe y Skourdoumbis, 2017; Solbrekke y Sugrue, 2014). De lo anterior, se espera que este conjunto de resultados e información pueda apoyar a entidades externas en la toma decisiones (Solbrekke y Sugrue, 2014), permitiendo legitimar e informar sobre la calidad y sobre sus procesos internos a la opinión pública (Solbrekke y Sugrue, 2014). En particular, en el caso de la formación de profesores, las instituciones deben contar con mecanismos de control que certifiquen los conocimientos, disposiciones y habilidades necesarios para enseñar y lograr aprendizajes en niños y niñas en el aula escolar (Talbot, 2016), lo que conlleva la supervision del contenido de las asignaturas en dichos programas de formación (Ling, 2017), diseñando marcos conceptuales para todos los programas, evaluando a los candidatos a docentes de dichas asignaturas, y creando sistemas de recopilación de datos para mejorar los aprendizajes profesionales de los egresados (Bell y Youngs, 2011; Ingvarson et al., 2007; Klemenz et al., 2019).

Con la incorporación de estándares, los procesos de acreditación comienzan a solicitar evidencia de objetivos, conceptos, métodos, fundamentos y medidas de evaluación en ámbitos del conocimiento profesional que deben alcanzar los estudiantes de pedagogía, el aseguramiento de espacios clínicos, la evaluación de la calidad del proceso y de los egresados de los programas de FID, junto a la determinación de procesos de retroalimentación (Deskriptif et al., 2012; Ling, 2017).

\section{OTROS ANTECEDENTES}

Para comprender este estudio es necesario abordar los siguientes apartados: i) Procesos de acreditación en la formación inicial de profesores, y ii) Características del proceso de acreditación en Chile, los cuales se describen a continuación.

\section{Procesos de acreditación en la formación inicial de profesores}

Con la incorporación de los estándares, el proceso de acreditación pone especial atención a la verificación de la eficacia del proceso de FID, ya que existe evidencia que aquellos profesores que pasaron por procesos de formación donde se evaluaron estas características mostraron estar mejor preparados para enseñar (DarlingHammond, 2020; Deskriptif et al., 2012). Por tal razón, se pide dentro del proceso de acreditación, verificar que existan cursos o asignaturas en los planes de estudio donde los estudiantes de pedagogía vivencien y experimenten modelos que se espera que ellos desarrollen con sus estudiantes (Ingvarson et al., 2007; Klemenz et al., 2019), verificando las metodologías en los procesos de enseñanza, las evaluaciones y el 
apoyo pedagógico a los estudiantes. En comparación con la orientación teórica de los cursos, este tipo actividades logra mayor eficacia de los estudiantes de pedagogía en su primer año de ejercicio (Klemenz et al., 2019). Conjuntamente con ello, se solicita evaluar los conocimientos profesionales de la disciplina y del contenido pedagógico mediante la observación del aula escolar, contrastando la información vía entrevistas, cuestionarios y analizando las producciones de los futuros profesores, o bien implementando procesos de valor agregado (Darling-Hammond, 2020; Ingvarson et al., 2007).

En esta línea, Ingvarson y sus colegas (2007) afirman que los procesos de acreditación donde sólo se leen las descripciones de los cursos de la FID, se visitan las instituciones y entrevistan al personal proporcionan poca evidencia y certeza de que los egresados del programa tengan realmente el conocimiento necesario y suficiente para usarlo en contextos reales, lo cual confirma la escasa validez y confiabilidad de estos procesos. Otro efecto negativo asociado a los procesos de acreditación es la asignación de la misma en una escala discreta, lo cual produce una clasificación informal de ránquines, originando comparaciones para identificar debilidades y fortalezas de los programas de FID (Bell y Youngs, 2011). Esta clasificación puede ser útil al informar a la opinión pública sobre la calidad de los procesos internos, orientando la contratación de profesionales y con ello determinar en parte las preferencias formativas de los jóvenes que quieren ingresar a la educación superior. No obstante, si las diferencias de clasificación no son significativas y claras entre los programas, la credibilidad, validez y confiabilidad de los datos que produce la acreditación se verían afectados (Bell y Youngs, 2011).

\section{Características del proceso de acreditación en Chile}

Chile en el año 2006 establece el proceso de acreditación obligatoria como el principal medio para demostrar la calidad de la FID, y así asegurar la eficacia de su sistema educativo (Domínguez y Meckes, 2011; Placier et al., 2016). Sin embargo, y en línea con el interés y cuestionamiento internacional, la acreditación en Chile ha sido enjuiciada por el exceso de responsabilidad que recae en jueces y pares evaluadores respecto de la asignación de estados de acreditación (Domínguez y Meckes, 2011; Placier et al., 2016). Esto afecta la confiabilidad y validez de la clasificación de los programas, dado que es una de las principales fuentes de error de cualquier proceso de evaluación (AERA, APA y NCME, 2014).

El proceso de acreditación actualmente vigente en Chile se desarrolla en tres etapas: a) autoevaluación de los programas y reporte de evidencias; b) evaluación externa por comisión de pares (jueces observadores); c) evaluación de procesos internos y externos reportados por un consejo de acreditación, los cuales aseguran la calidad del proceso de formación determinando el nivel de coherencia entre lo que se reporta en el programa y lo que se logra levantar en las tres etapas. En el año 2016 con la promulgación de la Ley que establece el Sistema Profesional Docente, la responsabilidad de acreditar los programas FID pasa de forma exclusiva al Consejo Nacional de Acreditación (CNA) (CNA, 2017) y dicho proceso evalúa el cumplimiento de tres dimensiones. Primero, la dimensión de Propósitos e Institucionalidad de la carrera o programa, donde se evalúan cinco criterios, a saber: propósitos, integridad, perfil de egreso, plan de estudios y vinculación con el medio. En segundo lugar, la dimensión de Condiciones de Operación, la que evalúa los criterios de Organización y administración, personal docente, infraestructura y recursos para el aprendizaje, participación y bienestar estudiantil, y creación e investigación por el cuerpo docente. Finalmente, la dimensión de Resultados y capacidad de autorregulación, que evalúa los criterios de efectividad y resultados del proceso formativo y autorregulación y mejoramiento continuo. Estos criterios se transforman en una rúbrica de evaluación para cada programa, tanto para el proceso de auto-reporte como para el proceso de evaluación de pares. Al finalizar el proceso, la acreditación asigna estados entre 0 y 7 años, los cuáles se fijan considerando la coherencia de los informes de autoevaluación, las evidencias cualitativas y cuantitativas reportadas, y el reporte de los pares evaluadores, más el juicio del presidente del CNA. Actualmente se exige a los programas de FID contar con la acreditación para acceder a los beneficios que entrega el Estado a los estudiantes de pedagogía y a las mismas instituciones.

Ahora bien, y en línea con lo que evalúan y observan los procesos de aseguramiento de los sistemas educativos exitosos y rigurosos, los programas de FID de calidad procuran dar cierto tipo de oportunidades de aprendizaje a los futuros profesores, las cuales se pueden organizar en dos focos: aquellas que brindan oportunidades de aprender la disciplina tal como se espera que sus alumnos las aprendan, generando así procesos de modelación de la práctica de enseñanza coherente con lo deseado (Ingvarson y Rowley, 2017). Otras, que brindan oportunidades de aprender a enseñar el contenido (planificar la enseñanza, analizar la gestión a través de episodios de aula, trabajar a partir de producciones de los alumnos, evaluar a través del levantamiento de evidencias de aprendizaje, entre otras), estableciendo una fuerte relación teoría-práctica, con experiencias clínicas supervisadas (Blömeke et al., 2015; Contreras, 2016; Klemenz et al., 2019; Werler y Tahirsylaj, 2020). Estos dos focos han demostrado tener impacto en el primer año de ejercicio del profesor, tanto en las prácticas de enseñanza como en el logro de aprendizajes de niños y niñas, adolescentes y estudiantes de pedagogía (Blömeke et al., 2015; Han et al., 2020; Ingvarson y Rowley, 2017; Lopez et al., 
2016), por lo que han sido incorporados como criterios de acreditación en referentes internacionales (Ingvarson y Rowley, 2017).

En función de los antecedentes anteriores, esta investigación explora cómo los programas FID de profesores de Educación Básica con diferentes años de acreditación, aproximan al aula escolar a este futuro profesor, para garantizar que se genere un proceso de calidad en el logro de aprendizaje asociado a la educación matemática (Kaiser, y König, 2019; Cerda et al., 2017). Esto se realiza a partir del examen de las oportunidades descritas en los syllabus de las asignaturas de Matemática y Didáctica de la Matemática que consideran los programas de formación de diversas instituciones de educación superior que imparten esta carrera.

Así, las preguntas que guían este estudio son: ¿Cuál es la presencia de las dimensiones de Conocimiento Matemático Escolar, Espacio, Rol y Actividad del Aula Escolar (Unidad CARE), extraídos a partir del análisis de las oportunidades descritas en los syllabus de las asignaturas de Matemática y Didáctica de la Matemática en las carreras de pedagogía de educación básica impartidas en Chile?, y sobre todo, ¿es posible observar una congruencia entre los indicadores de calidad CARE y los años de acreditación recibida por los programas examinados?

\section{MÉTODO}

El estudio se llevó a cabo en 15 de los 32 programas de formación de profesores de Educación Primaria de Chile del año 2017. Los programas analizados cuentan con 4, 5 y 6 años de acreditación, y pertenecen a 8 de las 15 regiones que tiene Chile (véase Tabla 1). A cada programa de formación que aceptó participar en la investigación, previa conformidad de confidencialidad y consentimientos de uso de la información, se le solicitó los syllabus de las asignaturas donde se abordaban los conocimientos matemáticos escolares y didáctico-matemáticos que los estudiantes de pedagogía en educación primaria debían conocer, según los lineamientos de los perfiles de egreso de cada uno de los programas de FID y establecidos en los planes de estudio. Cada programa de FID presentó en promedio $7.8(\mathrm{SD}=3.7)$ syllabus de asignaturas, con un mínimo de 3 y un máximo de 15. De lo anterior, el método de muestreo fue no probabilístico, por accesibilidad y voluntad de las instituciones formadoras a participar en la investigación.

Tabla 1: Región y asignaturas de los programas de formación inicial de la muestra.

\begin{tabular}{|c|c|c|c|}
\hline Programa FID & Años de acreditación & Región & № Asignaturas \\
\hline U1 & 6 & Metropolitana & 10 \\
\hline U2 & 6 & Bío Bío & 15 \\
\hline U3 & 6 & Metropolitana & 5 \\
\hline U4 & 6 & Bío Bío & 5 \\
\hline U5 & 6 & Araucanía & 10 \\
\hline U6 & 5 & Metropolitana & 4 \\
\hline U7 & 5 & Del Maule & 4 \\
\hline U8 & 5 & Bío Bío & 8 \\
\hline U9 & 5 & Aysén & 9 \\
\hline U10 & 5 & Arica y Parinacota & 9 \\
\hline U11 & 5 & Bío Bío & 4 \\
\hline U12 & 5 & Valparaíso & 3 \\
\hline U13 & 4 & Metropolitana & 13 \\
\hline U14 & 4 & Metropolitana & 12 \\
\hline U15 & 4 & Metropolitana & 7 \\
\hline \multicolumn{3}{|c|}{} & \\
\hline
\end{tabular}

\section{Etapas y propuestas de análisis estadísticos}

En una primera etapa, cada uno de los syllabus fue analizado mediante una codificación cualitativa deductiva (Mayring, 2015), formando categorías a partir de los nuevos paradigmas sobre la formación inicial que ponen el foco en la aproximación al aula escolar y en la resolución de situaciones de aula bajo el marco de la competencia profesional (Ver Figura 1) (Blömeke et al., 2020). En esta línea, la investigación (Blömeke et al., 2020; Grossman et al., 2009; Han et al. , 2020) demuestra que la "formación de la práctica" es un marco que permite al estudiante de pedagogía experimentar la realidad docente a través de representaciones, descomposiciones y aproximaciones al aula escolar. Esta aproximación a la realidad escolar, se lleva a cabo en cuatro dimensiones (Blömeke et al., 2020; DeGraff et al., 2015), las cuales son descritas a continuación: 
Conocimiento Matemático Escolar (CME): el proceso de formación debe aproximar el conocimiento disciplinar general que posee el estudiante de pedagogía al conocimiento escolar específico, profundo y profesional en educación matemática. Luego, y considerando las áreas del conocimiento matemático escolar, la dimensión CME, se desagrega en: Conocimiento Matemático No Escolar (CMNE), Conocimiento escolar de Números (N), Conocimiento escolar de Álgebra (A), Conocimiento escolar sobre Medida (M), Conocimiento escolar de Geometría (G), Conocimiento escolar de Datos y Azar (DA).

Espacio: el proceso de formación debe otorgar la oportunidad al estudiante de pedagogía vivenciar el espacio del aula escolar y todo lo que esto implica, como la interacción y comunicación con niños, niñas y colegas, gestionar el espacio y los recursos de la sala de clase, visualizar la disposición de los recursos, identificar posibles trayectorias de monitoreo, etc. Así, la aproximación en esta dimensión va desde el espacio del aula universitario al espacio del aula escolar. Para la codificación de esta dimensión se desagregó en Espacio de Aula escolar (EA) y en Espacio Universitario (EU).

Rol: La transición del rol de estudiante al rol de profesor es una de las oportunidades que la FID debe otorgar a los estudiantes de pedagogía. Para esto debe ofrecer oportunidades para la adquisición de la identidad de profesor por parte de los estudiantes de pedagogía. Esta dimensión se desagregó en Rol de Estudiante (RE) y en Rol de Profesor (RP).

Actividad del aula escolar (ACT): el proceso de formación debiera dar oportunidades a los estudiantes de pedagogía de hacer tareas que un profesor en ejercicio hace, lo que va desde la planificación de actividades a enfrentar situaciones de gestión de conducta. Dada la gran cantidad de actividades que puede realizar un profesor en el aula escolar, la dimensión ACT se desagrega en: Actividades de análisis Teórico (AT), Actividades de análisis de situaciones de Enseñanza general (AE), Actividades para el diseño de tareas Didáctico-matemáticas (AD); Actividades para la Implementación de tareas matemáticas (AI); Actividades para la Reflexión sobre producciones de tareas matemáticas ya implementadas (ARE). En conjunto estas dimensiones de aproximación al aula se denominan CARE, por las dimensiones Conocimiento, Actividad, Rol y Espacio. De esta forma, se considera a CARE como la unidad de comparación entre los programas FID con diferentes años de acreditación.

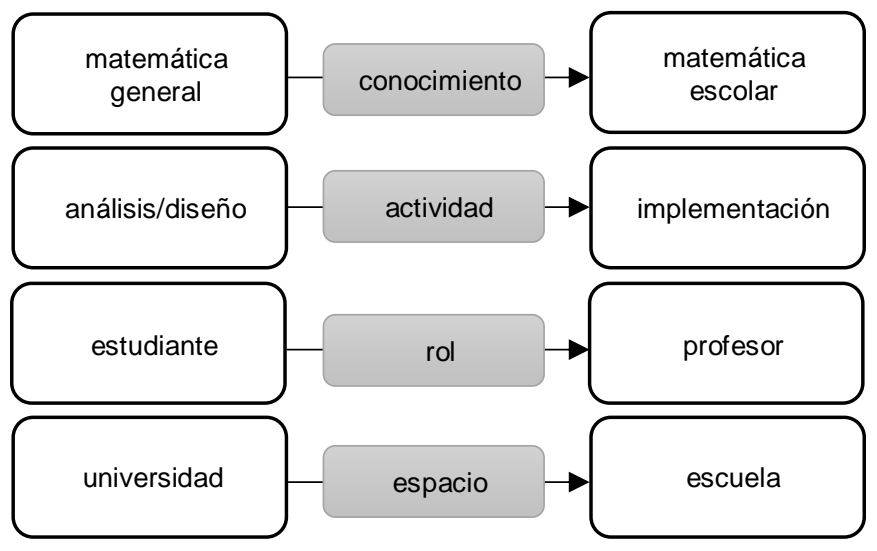

Fig. 1: Dimensiones de análisis cualitativo CARE

Cada uno de los syllabus fue codificado considerando como unidades de análisis las descripciones dispuestas en los apartados de objetivos, contenidos, metodologías y evaluaciones. El proceso de análisis de los syllabus se desarrolló en papel y digital, asignando los códigos CARE a cada uno de los apartados. La asignación se realizó mediante la observación de presencia o ausencia del código en la descripción del syllabus. En particular, las dimensiones sobre Espacio de aula y Actividades, se observaron en la metodología y en las evaluaciones descritas. Respecto del CME se observaron principalmente en los apartados de objetivos, contenidos y descripciones generales de las asignaturas. En cuanto a las actividades, estas se observaron en los apartados de objetivo, contenido y metodología. Lo anterior se fundamenta en que son los syllabus o programas de las asignaturas quienes direccionan el quehacer del formador en cada asignatura, entregando orientaciones sobre las oportunidades de aprendizaje que se deben dar en la FID (Darling-Hammond, 2017), metodología usada por diversos estudios para analizar y comparar los sistemas de formación de profesores (Werler y Tahirsylaj, 2020; Klemenz et al., 2019; Pushpanadham, 2020; CAEP, 2019). La codificación se realizó por dos correctores independientes, todas las dudas y discrepancias fueron revisadas por el investigador principal. El índice Kappa se calculó con el 100\% de los datos, los cuales variaron entre .91 y 1 con un promedio de $.94(\mathrm{SD}=0.17)$. 
En una primera etapa se realizó un análisis descriptivo con los códigos obtenidos del análisis cualitativo. Luego, se realizó un análisis de correspondencia con las dimensiones CARE. El análisis de correspondencia es un método inductivo que emplea gráficos e interpretaciones geométricas para explorar la estructura de relación de un conjunto de datos distribuidos en una matriz. Las relaciones se establecen entre las variables dispuestas en filas y columnas usando el test Chi cuadrado de Pearson $\chi^{2}$ de forma geométrica, es decir, la frecuencia de la presencia de los códigos en los syllabus se transforma en proporciones para obtener perfiles y centroídes (masas) para la clasificación de los datos. Esto se realiza para las filas, programas de FID, y lo mismo para las columnas (dimensiones CARE) (perfiles y centroídes). Así, el análisis permite distribuir los programas de FID en relación con las dimensiones CARE.

Los valores propios se calculan de modo que la primera dimensión explique la mayor varianza de la inercia total. El largo de los vectores permite ver la contribución de cada coordenada a la inercia de las dimensiones, en cuanto más largo sea el vector mayor contribución tendrá. Para su relación se deberá considerar el ángulo respecto de las dimensiones ortogonales. Por último, para asegurar la agrupación y las relaciones entre los programas de formación y las dimensiones de aproximación al aula escolar, se realizó un análisis jerárquico de clases con las coordenadas que arrojaba el análisis de correspondencia, lo que permitió agrupar a los programas de formación con relación a las dimensiones CARE (Hox et al., 2017). Todos los análisis se llevaron a cabo con el software RStudio usando los paquetes "FactoMiner" y las funciones "CA" (Kassambara, 2017).

\section{RESULTADOS}

Tal como se observa en la Tabla 2, la dimensión CME se observó entre 13 y 62 veces, con media 31.12 ( $S D=13.87)$ y los códigos relativos al CMNE entre 0 y 20 veces, con media $5.2(S D=5.76)$. Los códigos relativos a las ACT, las que se refieren a AT se observaron entre 0 y 11 veces, con media 4.33 ( $S D=3.35)$, los que se relacionaban a $A E$, se observaron entre 5 y 38 veces, con media $17.07(S D=10.17)$, los que se relacionaron con $A D$ se observaron entre 7 y 82 veces, con media $33.6(S D=19.44)$, los relativos a $A l$ se observan entre 2 y 29 veces, con media $12.33(S D=8.16)$, y relativos a ARE se observaron entre 0 y 18 veces, con media $7.87(\mathrm{SD}=5.74)$. Los códigos relativos a la dimensión $\mathrm{RE}$ se observaron entre 11 y 175 , con media $73.67(\mathrm{SD}=44.63)$, por otra parte, los códigos asociados al RP se observaron entre 0 y 129, con media 49.73 ( $\mathrm{SD}=43.40)$. Por último, los relativos a EU se observaron entre 11 y 175 veces, con media 72,27 ( $\mathrm{SD}=44.06)$. La sub-dimensión EA se observó en solo uno de los programas FID, observándose entre 0 y 18 veces, con media $7.4(S D=5.4)$.

Tabla 2: Frecuencia de indicadores de la unidad CARE observados en la descripción de los syllabus.

\begin{tabular}{|c|c|c|c|c|c|c|c|c|c|c|c|}
\hline & \multicolumn{2}{|c|}{ Conocimiento } & \multicolumn{5}{|c|}{ Actividad } & \multicolumn{2}{|c|}{ Rol } & \multicolumn{2}{|c|}{ Espacio } \\
\hline & $\begin{array}{c}M E \\
M \\
(S D)\end{array}$ & $\begin{array}{c}M N E \\
M \\
(S D)\end{array}$ & $\begin{array}{c}A T \\
M \\
(S D)\end{array}$ & $\begin{array}{c}A E \\
M \\
(S D)\end{array}$ & $\begin{array}{c}A D \\
M \\
(S D)\end{array}$ & $\begin{array}{c}A l \\
M \\
(S D)\end{array}$ & $\begin{array}{c}A R E \\
M \\
(S D)\end{array}$ & $\begin{array}{c}R E \\
M \\
(S D) \\
\end{array}$ & $\begin{array}{c}R P \\
M \\
(S D)\end{array}$ & $\begin{array}{c}E A \\
M \\
(S D)\end{array}$ & $\begin{array}{c}E U \\
M \\
(S D)\end{array}$ \\
\hline \multirow[t]{3}{*}{ U1 } & 56,0 & 8,0 & 0,0 & 26,0 & 40,0 & 22,0 & 12,0 & 100,0 & 75,0 & 79,0 & 56,0 \\
\hline & 5,6 & 0,8 & 0,0 & 2,6 & 4,0 & 2,2 & 1,2 & 10,0 & 7,5 & 7,9 & 5,6 \\
\hline & $(5,2)$ & $(1,3)$ & $(0,0)$ & $(2,3)$ & $(5,1)$ & $(3,0)$ & $(2,1)$ & $(11,6)$ & $(12,8)$ & $(11,3)$ & $(12,3)$ \\
\hline \multirow[t]{3}{*}{$\mathrm{U} 2$} & 62,0 & 20,0 & 8,0 & 38,0 & 82,0 & 29,0 & 18,0 & 175,0 & 121,0 & 175,0 & 0,0 \\
\hline & 6,2 & 2,0 & 0,8 & 3,8 & 8,2 & 2,9 & 1,8 & 17,5 & 12,1 & 17,5 & 0,0 \\
\hline & $(5,2)$ & $(2,3)$ & $(1,1)$ & $(3,6)$ & $(6,1)$ & $(2,7)$ & $(1,1)$ & $(13,9)$ & $(15,8)$ & $(13,9)$ & $(0,0)$ \\
\hline \multirow[t]{3}{*}{ U3 } & 31,0 & 2,0 & 3,0 & 16,0 & 50,0 & 14,0 & 3,0 & 86,0 & 64,0 & 86,0 & 0,0 \\
\hline & 3,1 & 0,2 & 0,3 & 1,6 & 5,0 & 1,4 & 0,3 & 8,6 & 6,4 & 8,6 & 0,0 \\
\hline & $(3,5)$ & $(0,4)$ & $(0,5)$ & $(2,1)$ & $(6,5)$ & $(2,3)$ & $(0,5)$ & $(10,9)$ & $(10,6)$ & $(10,9)$ & $(0,0)$ \\
\hline \multirow[t]{3}{*}{$\mathrm{U} 4$} & 21,0 & 4,0 & 4,0 & 10,0 & 26,0 & 9,0 & 6,0 & 55,0 & 19,0 & 55,0 & 0,0 \\
\hline & 2,1 & 0,4 & 0,4 & 1,0 & 2,6 & 0,9 & 0,6 & 5,5 & 1,9 & 5,5 & 0,0 \\
\hline & $(2,8)$ & $(0,7)$ & $(0,7)$ & $(1,6)$ & $(3,2)$ & $(1,3)$ & $(1,1)$ & $(7,1)$ & $(6,0)$ & $(7,1)$ & $(0,0)$ \\
\hline \multirow[t]{3}{*}{ U5 } & 46,0 & 15,0 & 9,0 & 25,0 & 30,0 & 12,0 & 10,0 & 86,0 & 51,0 & 86,0 & 0,0 \\
\hline & 4,6 & 1,5 & 0,9 & 2,5 & 3,0 & 1,2 & 1,0 & 8,6 & 5,1 & 8,6 & 0,0 \\
\hline & $(3,7)$ & $(2,5)$ & $(1,2)$ & $(2,1)$ & $(3,7)$ & $(1,9)$ & $(0,7)$ & $(8,7)$ & $(8,8)$ & $(8,7)$ & $(0,0)$ \\
\hline \multirow[t]{3}{*}{ U6 } & 22,0 & 2,0 & 2,0 & 7,0 & 38,0 & 13,0 & 1,0 & 61,0 & 0,0 & 61,0 & 0,0 \\
\hline & 2,2 & 0,2 & 0,2 & 0,7 & 3,8 & 1,3 & 0,1 & 6,1 & 0,0 & 6,1 & 0,0 \\
\hline & $(3,5)$ & $(0,6)$ & $(0,4)$ & $(0,9)$ & $(5,0)$ & $(2,1)$ & $(0,3)$ & $(8,0)$ & $(0,0)$ & $(8,0)$ & $(0,0)$ \\
\hline \multirow[t]{3}{*}{ U7 } & 17,0 & 0,0 & 6,0 & 17,0 & 22,0 & 10,0 & 6,0 & 61,0 & 55,0 & 61,0 & 0,0 \\
\hline & 1,7 & 0,0 & 0,6 & 1,7 & 2,2 & 1,0 & 0,6 & 6,1 & 5,5 & 6,1 & 0,0 \\
\hline & $(3,6)$ & $(0,0)$ & $(1,3)$ & $(3,6)$ & $(4,6)$ & $(2,2)$ & $(1,3)$ & $\begin{array}{l}(12,9) \\
\end{array}$ & $(11,6)$ & $\begin{array}{l}(12,9) \\
\end{array}$ & $(0,0)$ \\
\hline
\end{tabular}


Tabla 2: continuación

\begin{tabular}{|c|c|c|c|c|c|c|c|c|c|c|c|}
\hline & \multicolumn{2}{|c|}{ Conocimiento } & \multicolumn{5}{|c|}{ Actividad } & \multicolumn{2}{|c|}{ Rol } & \multicolumn{2}{|c|}{ Espacio } \\
\hline & $\begin{array}{c}M E \\
M \\
(S D)\end{array}$ & $\begin{array}{c}M N E \\
M \\
(S D)\end{array}$ & $\begin{array}{c}A T \\
M \\
(S D)\end{array}$ & $\begin{array}{c}A E \\
M \\
(S D)\end{array}$ & $\begin{array}{c}A D \\
M \\
(S D)\end{array}$ & $\begin{array}{c}A I \\
M \\
(S D)\end{array}$ & $\begin{array}{c}A R E \\
M \\
(S D)\end{array}$ & $\begin{array}{c}R E \\
M \\
(S D)\end{array}$ & $\begin{array}{c}R P \\
M \\
(S D)\end{array}$ & $\begin{array}{c}E A \\
M \\
(S D)\end{array}$ & $\begin{array}{c}E U \\
M \\
(S D)\end{array}$ \\
\hline \multirow[t]{3}{*}{ U8 } & 31,0 & 1,0 & 1,0 & 15,0 & 39,0 & 9,0 & 15,0 & 79,0 & 54,0 & 79,0 & 0,0 \\
\hline & 3,1 & 0,1 & 0,1 & 1,5 & 3,9 & 0,9 & 1,5 & 7,9 & 5,4 & 7,9 & 0,0 \\
\hline & $(3,4)$ & $(0,3)$ & $(0,3)$ & $(1,4)$ & $(3,6)$ & $(1,1)$ & $(1,6)$ & $(7,5)$ & $(7,1)$ & $(7,5)$ & $(0,0)$ \\
\hline \multirow[t]{3}{*}{ U9 } & 25,0 & 3,0 & 3,0 & 5,0 & 16,0 & 5,0 & 3,0 & 17,0 & 4,0 & 17,0 & 0,0 \\
\hline & 2,5 & 0,3 & 0,3 & 0,5 & 1,6 & 0,5 & 0,3 & 1,7 & 0,4 & 1,7 & 0,0 \\
\hline & $(2,4)$ & $(0,7)$ & $(0,5)$ & $(0,8)$ & $(1,9)$ & $(0,8)$ & $(0,7)$ & $(2,9)$ & $(1,0)$ & $(2,9)$ & $(0,0)$ \\
\hline \multirow[t]{3}{*}{ U10 } & 30,0 & 3,0 & 0,0 & 6,0 & 10,0 & 2,0 & 0,0 & 11,0 & 0,0 & 11,0 & 0,0 \\
\hline & 3,0 & 0,3 & 0,0 & 0,6 & 1,0 & 0,2 & 0,0 & 1,1 & 0,0 & 1,1 & 0,0 \\
\hline & $(3,3)$ & $(0,7)$ & $(0,0)$ & $(1,1)$ & $(1,2)$ & $(0,4)$ & $(0,0)$ & $(2,2)$ & $(0,0)$ & $(2,2)$ & $(0,0)$ \\
\hline \multirow[t]{3}{*}{ U11 } & 21,0 & 0,0 & 1,0 & 9,0 & 7,0 & 2,0 & 4,0 & 23,0 & 0,0 & 23,0 & 0,0 \\
\hline & 2,1 & 0,0 & 0,1 & 0,9 & 0,7 & 0,2 & 0,4 & 2,3 & 0,0 & 2,3 & 0,0 \\
\hline & $(3,4)$ & $(0,0)$ & $(0,3)$ & $(1,9)$ & $(1,3)$ & $(0,4)$ & $(0,5)$ & $(3,6)$ & $(0,0)$ & $(3,6)$ & $(0,0)$ \\
\hline \multirow[t]{3}{*}{ U12 } & 13,0 & 2,0 & 6,0 & 10,0 & 13,0 & 4,0 & 4,0 & 36,0 & 10,0 & 36,0 & 0,0 \\
\hline & 1,3 & 0,2 & 0,6 & 1,0 & 1,3 & 0,4 & 0,4 & 3,6 & 1,0 & 3,6 & 0,0 \\
\hline & $(3,2)$ & $(0,6)$ & $(1,6)$ & $(1,6)$ & $(2,2)$ & $(1,0)$ & $(0,5)$ & $(5,8)$ & $(3,2)$ & $(5,8)$ & $(0,0)$ \\
\hline \multirow[t]{3}{*}{ U13 } & 36,0 & 10,0 & 11,0 & 35,0 & 51,0 & 26,0 & 17,0 & 140,0 & 129,0 & 140,0 & 0,0 \\
\hline & 3,6 & 1,0 & 1,1 & 3,5 & 5,1 & 2,6 & 1,7 & 14,0 & 12,9 & 14,0 & 0,0 \\
\hline & $(4,1)$ & $(1,6)$ & $(1,2)$ & $(2,9)$ & $(5,7)$ & $(3,6)$ & $(2,4)$ & $(13,8)$ & $(13,3)$ & $(13,8)$ & $(0,0)$ \\
\hline \multirow[t]{3}{*}{ U14 } & 27,0 & 5,0 & 5,0 & 19,0 & 39,0 & 13,0 & 11,0 & 87,0 & 82,0 & 87,0 & 0,0 \\
\hline & 2,7 & 0,5 & 0,5 & 1,9 & 3,9 & 1,3 & 1,1 & 8,7 & 8,2 & 8,7 & 0,0 \\
\hline & $(3,0)$ & $(0,7)$ & $(0,8)$ & $(2,3)$ & $(3,8)$ & $(1,4)$ & $(1,2)$ & $(8,8)$ & $(8,4)$ & $(8,8)$ & $(0,0)$ \\
\hline \multirow[t]{3}{*}{ U15 } & 29,0 & 3,0 & 6,0 & 18,0 & 41,0 & 15,0 & 8,0 & 88,0 & 82,0 & 88,0 & 0,0 \\
\hline & 2,9 & 0,3 & 0,6 & 1,8 & 4,1 & 1,5 & 0,8 & 8,8 & 8,2 & 8,8 & 0,0 \\
\hline & $(3,8)$ & $(0,5)$ & $(1,1)$ & $(2,7)$ & $(7,4)$ & $(2,8)$ & $(1,2)$ & $(14,8)$ & $(13,8)$ & $(14,8)$ & $(0,0)$ \\
\hline
\end{tabular}

En la Tabla 2, el significado de los símbolos y abreviaturas es el siguiente: $\mathrm{M}=$ Promedio de indicadores observados en 10 semestres; SD=Desviación Estándar de indicadores observados en 10 semestres; $\mathrm{ME}=$ Matemática Escolar; $\mathrm{MNE}=$ Matemática No Escolar; $\mathrm{AT}=$ Actividades de Análisis Teórico; $\mathrm{AE}=\mathrm{Actividades}$

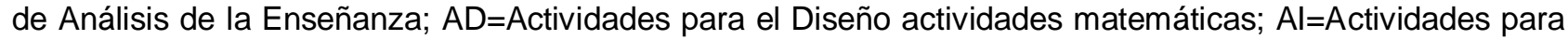
la Implementación de actividades matemáticas; ARE=Actividades para Reflexionar sobre resultados de implementación; RE=Rol como Estudiante; RP=Rol como Profesor; EU=Actividades realizadas en el Espacio Universitario; EA=Actividades realizadas en el Espacio del Aula escolar.

\section{FID, dimensiones CARE y años de acreditación}

A continuación, se muestran relaciones de correspondencia entre los programas FID identificados con los años de acreditación y las dimensiones CARE. En un segundo apartado se observa la correspondencia entre los programas FID identificados con los años de acreditación con las sub-dimensiones de CARE: Conocimiento matemático escolar y Tipo de Actividades.

La correspondencia entre los programas FID con 4, 5 y 6 años de acreditación y las variables CARE es alta y significativa $\chi^{2}(6, \mathrm{~N}=1992)=74.25, \mathrm{p}<.000$, reportando sólo dos valores propios, los cuales explican el $100 \%$ de la varianza acumulada de la inercia total. La varianza explicada por la primera dimensión es de $86.89 \%$, y el resto se asigna a la segunda. Como se observa en la Figura 2, CME y ACT se relacionan con programas que tienen 5 años de acreditación entorno a la primera dimensión. En el lado opuesto se encuentran los programas que tienen 6 años de acreditación, los cuales se relacionan con la variable EA y RP. En la segunda dimensión, los programas con 4 años de acreditación se relacionan con programas donde se observa la asignación de RP a los estudiantes de pedagogía. En el lado negativo, la dimensión 2 se caracteriza por la relación entre los programas que tienen 5 años de acreditación y las variables ACT y CME. 


\section{FID, Conocimiento Matemático y años de acreditación}

La correspondencia entre los programas FID y las variables del conocimiento matemático es alta y significativa respecto de los años de acreditación $\chi^{2}(10, N=569)=23.72, p<.000$ reportando sólo dos valores propios, los cuales explican el $100 \%$ de la varianza acumulada de la Inercia total. La varianza explicada por la primera dimensión es de $64.34 \%$, y el resto es explicada por la segunda. Como se observa en la Figura 3 , los programas con 5 años de acreditación se relacionan con las variables de conocimiento matemático escolar de Números entorno a la primera dimensión.

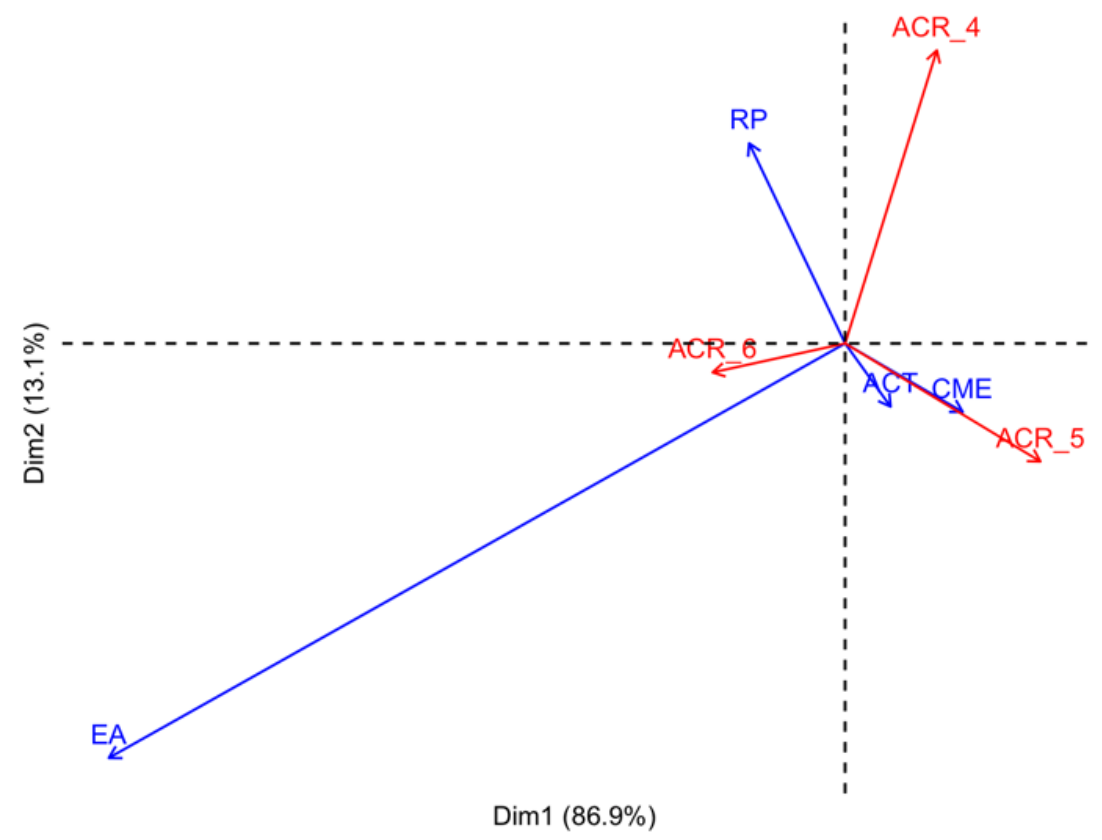

Fig. 2. Análisis de correspondencia entre los programas de FID de Educación Básica de Chile y las variables CARE de aproximación al aula escolar.

En el lado opuesto a esta dimensión se encuentran los programas que tienen 6 años de acreditación, los cuales se relacionan con las variables de Conocimiento Matemático No Escolar. En la segunda dimensión, los programas con 4 años de acreditación se relacionan con las variables de conocimiento escolar de Álgebra. En lado negativo, la dimensión 2 se caracteriza por la relación entre los programas que tienen 6 años de acreditación y las variables de conocimiento escolar de Geometría. Las nomenclaturas para comprender la Figura 2 y 3 son las siguientes: ACR 4=Programas FID con 4 años de acreditación; ACR 5=Programas FID con 5 años de acreditación; ACR $\overline{6}=$ Programas FID con 6 años de acreditación; CMNE=Conocimiento

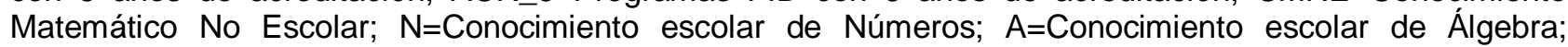
$\mathrm{M}=$ Conocimiento escolar sobre Medida; $\mathrm{G}=$ Conocimiento escolar de Geometría; $\mathrm{DA}=$ Conocimiento escolar de Datos y Azar; $\mathrm{CME}=$ Conocimiento Matemático Escolar; RP=Rol de Profesor; $\mathrm{ACT}=$ Actividades desarrolladas en el aula escolar.

\section{FID, Actividades CARE y años de acreditación}

La correspondencia entre los años de acreditación y las actividades descritas a desarrollar en las asignaturas de Matemática y Didáctica de la Matemática no es significativa $\left(\chi^{2}(8, N=1128)=4.26, p=.83\right)$ reportando sólo dos valores propios, los cuales explican el $100 \%$ de la varianza acumulada de la Inercia total. La varianza explicada por la primera dimensión es de $65.17 \%$, y el resto se asigna a la segunda. Como se observa en la Figura 4 actividades de análisis teórico (AT) se relacionan con programas que tienen 4 años de acreditación entorno a la primera dimensión y en menor medida actividades de reflexión sobre producciones de tareas matemáticas, en el lado opuesto se encuentran los programas que tienen 6 años de acreditación, los cuales se relacionan con actividades enfocadas al diseño de tareas matemáticas (AD). En la segunda dimensión, los programas con 5 años de acreditación se relacionan con actividades con foco en el diseño de tareas matemáticas (AD). En cuadrante negativo, la dimensión 2 se caracteriza por la relación entre los programas que tienen 6 años de acreditación y las actividades con foco en la implementación (Al) de Tareas Matemáticas.

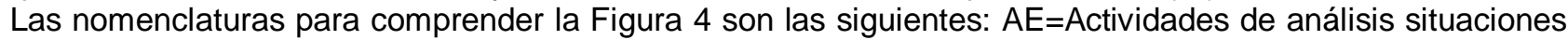
Enseñanza General; ARE=Actividades para la reflexión sobre producciones de tareas matemáticas implementadas; ACR_4=Programas FID con 4 años de acreditación; ACR_5=Programas FID con 5 años de acreditación; ACR_6=Programas FID con 6 años de acreditación. 


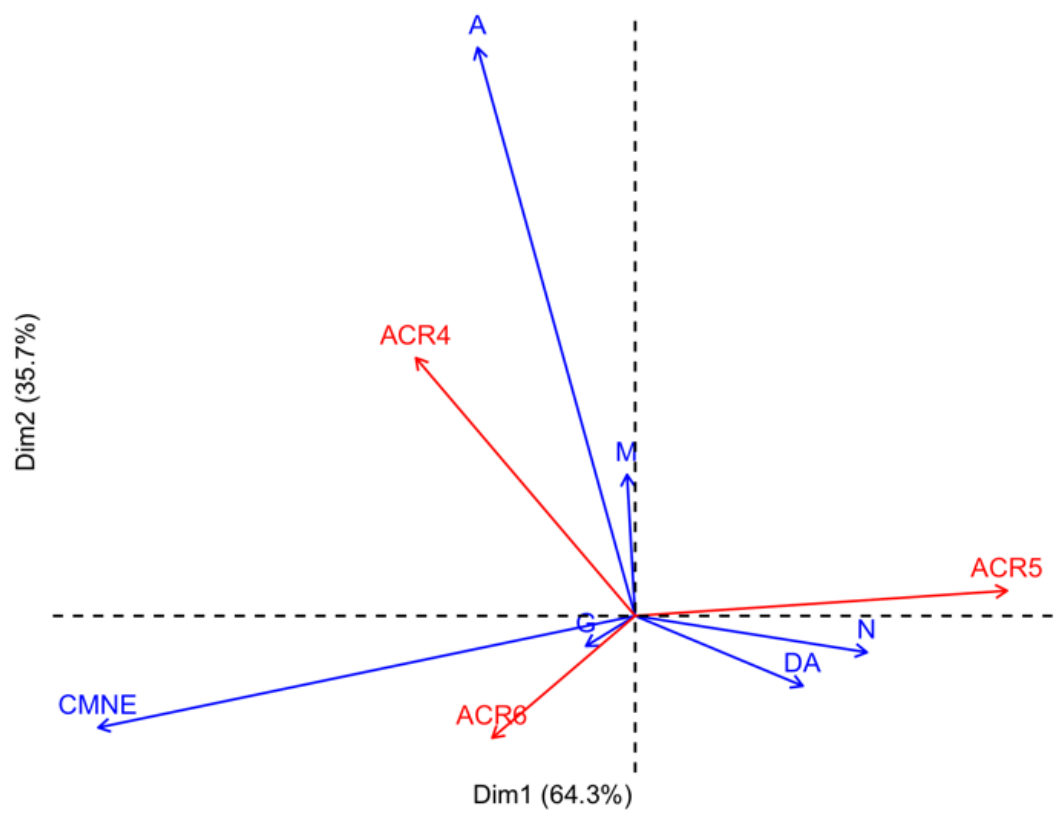

Fig. 3. Correspondencia entre programas FID, Conocimiento Matemático CARE, y años de acreditación.

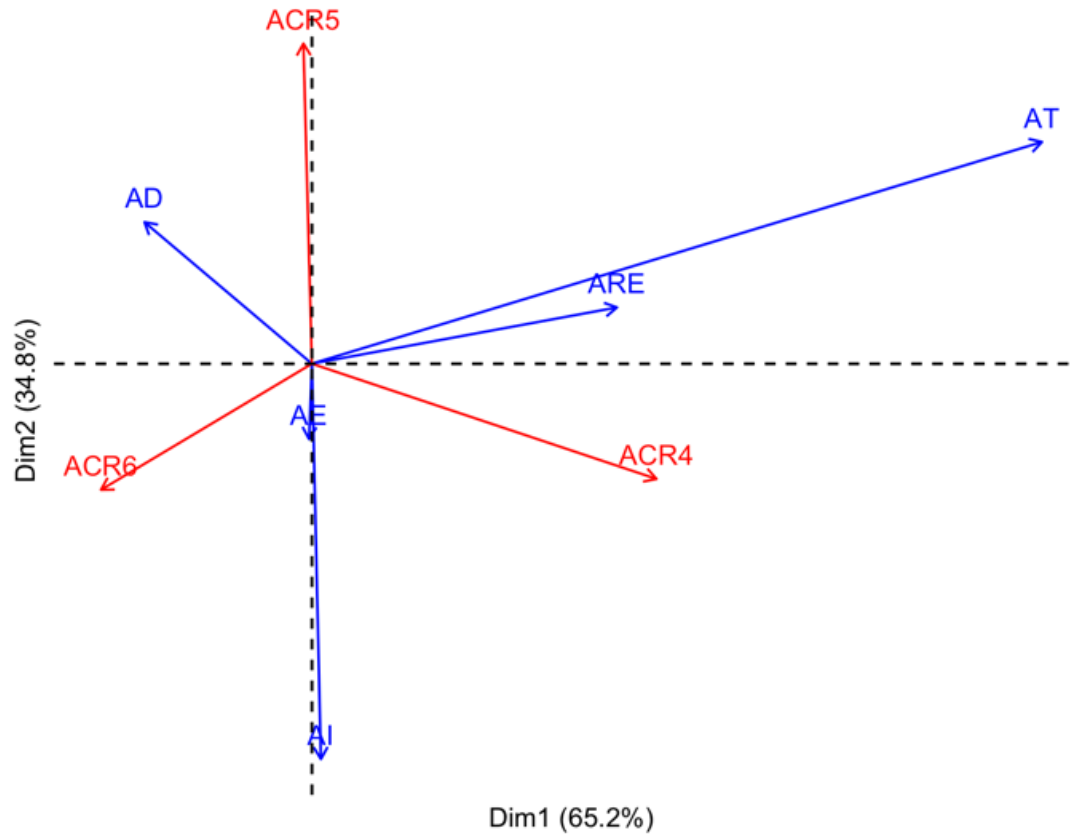

Fig. 4. Correspondencia entre los programas FID de Educación Básica de Chile, las variables de Actividades CARE y sus años de acreditación.

\section{DISCUSIÓN}

En función de las preguntas de investigación, los hallazgos del presente estudio establecen algunas características diferenciales de la presencia de las dimensiones de la unidad CARE en sus dimensiones de Conocimiento Matemático Escolar, Espacio, Rol y Actividad del Aula Escolar, extraídos de las oportunidades descritas en los syllabus de las asignaturas de Matemática y Didáctica de la Matemática y analizados respecto de los años de acreditación de los diversos programas de las carreras de pedagogía básica examinadas. Dentro de las características posibles de inferir, se puede sintetizar lo siguiente: 
Los programas con 4 años de acreditación se caracterizan por realizar actividades donde se le asigna a los estudiantes de pedagogía el rol de profesor, como simulaciones de clases escolar en el aula universitaria. Estas actividades se diseñan por los propios estudiantes de pedagogía quienes integran aspectos curriculares, teóricos, didácticos, y conocimientos matemáticos escolares. Los EP toman decisiones tal como si éstos estuvieran en el aula escolar, respondiendo a las acciones o situaciones de contingencia, realizando procesos de reflexión pedagógica en situ o posteriormente a la implementación de la actividad. Típicamente se usan como herramientas de evaluación.

En cuanto al tipo de conocimiento, se aborda con mayor frecuencia conocimientos escolares relativos al área de Álgebra: Variables y expresiones algebraicas, identidades, ecuaciones, resolución de ecuaciones, desigualdades e inecuaciones, sistema de ecuaciones de primer grado, patrones, sucesiones, funciones, proporciones, porcentajes, etc. Y en el área de Medida: determinación de la longitud, perímetro, área y volumen, uso de unidades no estandarizadas y estandarizadas, y la unidad de tiempo (en escasas ocasiones). Respecto a las actividades, estos programas se caracterizaron por abordar actividades de: análisis de teorías didácticas generales o ligadas a la enseñanza de la matemática; de análisis epistemológico o histórico de las matemáticas; de análisis sobre teorías del aprendizajes o del desarrollo humanos; de análisis de resultados de actividades ya implementadas tales como videos de clases, análisis de resultados de evaluaciones, respuestas de niños y niñas, efectos de orientaciones o preguntas de profesores.

Por otro lado, aquellos programas con 5 años de acreditación se caracterizaron por solicitar el desarrollo de actividades de un profesor en ejercicio, tales como: diseñar tareas matemáticas, evaluaciones diagnósticas y de formación, seleccionar contenidos y secuenciarlos, buscar, analizar y seleccionar metodologías de enseñanza, analizar recursos didácticos, observar, analizar y reflexionar sobre situaciones enseñanza registradas en casos escritos, grabaciones de audio o en video de clases, estudiar textos escolares, etc. Estas características estaban descritas principalmente en la descripción metodológica del syllabus. También, se caracterizan por abordar conocimiento matemático escolar en las áreas de Números, Álgebra, Geometría y Datos y Azar, alcanzando una mayor frecuencia el eje de Números, donde se describían contenidos como: el conjunto de los números naturales, enteros, racionales e irracionales, sistemas de numeración decimal, sistemas posicionales, aditivos o relativos, razones, proporcionalidad, y con menor frecuencia, contenidos relativos a teoría de números, como divisibilidad, números primos, descomposición prima, máximo común divisor y mínimo común múltiplo. Asimismo, los conocimientos relativos a Datos y Azar observados fueron: muestreo, tipos de variables, representación y organización de datos en tablas y gráficos para variables cualitativas como gráficos concretos, pictogramas y de barra, y gráficos para variables cuantitativas como gráficos de puntos, de línea y de dispersión, aunque en menor frecuencia que el eje de Números.

En muy pocos syllabus se observaron análisis y establecimiento de inferencias o correlaciones, o contenidos relacionados a probabilidades, como lenguaje probabilístico, azar, incertidumbre, probabilidad subjetiva, axiomática y frecuentista. Respecto de los tipos de actividades, estos programas se caracterizaron por solicitar a los estudiantes de pedagogía diseñar situaciones de clase, tales como: análisis, selección y diseño de actividades de clases, secuenciación de tareas matemáticas para su implementación en clases de 90 minutos, secuenciación de unidades semestrales o anuales, diseño de evaluaciones diagnósticas y formativas.

Finalmente, los programas con 6 años de acreditación se caracterizaban por realizar actividades donde confluyen el rol de profesor, las actividades y el conocimiento matemático escolar, tales como observación directa de clases, respuestas o reacciones de los estudiantes, implementaciones de planificaciones de clase elaboradas en el espacio universitario, análisis, reflexión y toma de decisiones in situ, implementación de evaluaciones, reflexiones compartidas con colegas sobre situaciones vividas en el aula escolar y atención de apoderados en relación a procesos de evaluación. Si bien estos programas abordaban conocimiento matemático escolar, como por ejemplo en Geometría con contenidos relativos a la Geometría Euclidiana, Transformaciones geométricas, Congruencia y Semejanza, Construcción con regla y compas, Geometría en 3D como vistas de cuerpos, redes y posiciones relativas, su principal característica son contenidos matemáticos no escolares. Aunque algunos puedan comprenderse en el horizonte la matemática escolar, se observan muchos temas cuyo alcance excede el campo de conocimiento necesario para un profesor de Educación Primaria, como por ejemplo, los conocimientos de Números, teoría de conjuntos, teorema fundamental de la aritmética, ecuaciones diofánticas, matemática discreta, lógica matemática y métodos numéricos.

En el caso de Geometría, Geometría axiomática, analítica y de coordenadas; en Álgebra, el teorema fundamental del álgebra, análisis elemental y polinomios; en Estadística y Probabilidades, Inferencia Estadística y procesos Estocásticos, además de algunos contenidos marginalmente considerados, tales como teoría de grupos, anillos, campos e ideales, Geometrías No-Euclidianas y Topología. Respecto de las actividades, se caracterizan por solicitar análisis de procesos y teorías de enseñanza en relación a teorías 
curriculares, rol del profesor, fines del sistema escolar, relaciones profesor - alumno, desarrollo profesional docente, diseño instruccional, uso de TIC y otros medios para apoyar la enseñanza, desarrollo de conceptos matemáticos, razonamiento y argumentación matemática, desarrollo de procedimientos, conocimiento práctico, gestión de la enseñanza para alumnos con diversas habilidades y estilos de aprendizaje, colaboración entre profesores, análisis teórico de elaboración de planes de clase, teoría de gestión del aula, teorías sobre el desarrollo de ambientes de aprendizaje y manejo del comportamiento inapropiado.

De lo anterior, se constata que la dimensión Conocimiento Matemático de la unidad CARE es la única que permite observar una diferencia entre los programas de FID con diferentes años de acreditación. Sin embargo, esta diferencia no es anidada, en sentido que los conocimientos y/o habilidades de un programa de 6 años no contienen, necesariamente, a los conocimientos de un programa de 4 ó 5 años de acreditación. Esto parece contraproducente con los lineamientos de los procesos de acreditación, los que en su primera dimensión de criterios (CNA, 2017), establecen y verifican que los programas aborden los conocimientos y habilidades necesarios para un proceso de FID Educación Básica en cualquiera de sus áreas disciplinares, tal como se observa en los criterios 3.d y 4.b de la Resolución Exenta DJ 009-4 de la CNA (2017). En el resto de las dimensiones CARE los programas no presentan diferencias significativas. Por ejemplo y en relación a la dimensión de Actividades, los programas describen abordar el mismo tipo de actividades como análisis, diseño, implementación y reflexión de situaciones de enseñanza y aprendizaje de las matemáticas.

En relación a la dimensión Rol, los programas suelen mantener el papel de estudiante hasta que ingresan a las asignaturas de prácticas progresivas, las cuales no están asociados necesariamente a la enseñanza 0 aprendizaje de la matemática. Destacan los programas U1 y U7, con 5 y 6 años de acreditación y los programas con cuatro 4 años de acreditación quienes en las asignaturas de didáctica de las matemáticas declaran desarrollar actividades de simulación de clases donde se les asigna el rol de Profesor. Por último, la dimensión Espacio es la que comparten todos los programas, desarrollando las actividades de formación principalmente en el aula universitaria. Sólo uno de los programas plantea realizar actividades de formación en el aula escolar y no en el universitario.

Si el único medio relevante para verificar la calidad de un sistema de formación de profesores es la acreditación, este debe ser responsable de su calidad, validez y confiabilidad de sus resultados (Ling, 2017). Si bien en Chile, los procesos de acreditación aún no responden a la verificación de estándares del proceso de formación para la verificación de conocimientos y habilidades de los egresados de los programas (Ingvarson et al., 2007; Ingvarson y Rowley, 2017; Mayer et al., 2017), los resultados de este estudio la convierten en una necesidad (Talbot, 2016), dado que la medición de la calidad por medio de la coherencia interna del proceso de formación muestra no ser real. Así, la política educativa de aseguramiento de la calidad debe avanzar desde la coherencia de los procesos de FID a la muestra de evidencia de efectividad de los egresados en el aula escolar (Deskriptif et al., 2012; Ingvarson et al., 2007; Klemenz et al., 2019). Partiendo de la base que los cursos de didáctica y de matemática son cursos centrales en la formación de un profesor de Enseñanza Básica, se podría justificar que a través del análisis de sus syllabus, se pueden vislumbrar algunos aspectos del juicio evaluativo de la acreditación, ya que en esta descripción, afloran elementos de vinculación con el medio (redes de establecimientos para las actividades), propósitos (finalidad de las actividades respecto del perfil de egreso), condiciones de operación (para coordinar las actividades de estas asignaturas con el resto de la malla), recursos (traslados, facilidades), efectividad (presencia en el perfil) y autorregulación (cómo estas acciones son tomadas para retroalimentar y mejorar la formación).

Los resultados muestran que la dimensión de Conocimiento Matemático Escolar es la dimensión de aproximación al aula escolar que se observa de forma transversal en los programas de formación analizados. El resto de las dimensiones de CARE tiene una presencia variable entre programas con diferente e igual cantidad de años de acreditación. Aunque algunos programas destacan en la dimensión de Espacio el dar oportunidades, en las mismas asignaturas de Matemática o Didáctica la Matemática, de desarrollar actividades en el espacio del aula escolar y otros que promueven el Rol de profesor en el espacio de aula Universitario. Respecto de las Actividades, los programas con más años presentan mayor relación al quehacer de un profesor en el aula.

Así, pareciera que la asignación de diferentes estados de acreditación no está asegurando una mejor o peor calidad de los programas FID, ya que la jerarquía como resultado secundario de la escala no tiene significado, provocando incertidumbre en su interpretación. Se podría pensar que tener más años de acreditación asegura el cumplimiento de aspectos que un programa con menos años no logra. Sin embargo, los resultados no muestran este anidamiento en las características establecidas por CARE entre los diferentes tipos de programa. De esta forma, mediante este estudio se concluye que no es posible establecer una diferencia en relación a qué conocimientos y habilidades obtiene un profesor egresado de un programa de formación u otro medidos en años de acreditación, en cuanto a la aproximación al aula escolar de educación matemática que esta le pueda entregar (Solbrekke y Sugrue, 2014). 
Si bien las oportunidades descritas en la unidad de análisis del estudio, los syllabus de las asignaturas de Matemática y Didáctica de las Matemáticas, pueden ser las esperadas a dar en los procesos de formación de profesores, condiciones como las capacidades del formador para interpretarlas e implementarlas, el conocimiento y hábitos de aprendizaje de los estudiantes de pedagogía, los recursos de las instituciones, etc. podrían distanciar la esperanza de lo que realmente ocurre en el aula de formación inicial. Esta limitación metodológica permite aseverar que todas las afirmaciones del presente estudio son potenciales, por un lado, pero por otro, incrementan las diferencias observadas entre los procesos de formación de programas con diferentes años de acreditación dada la distancia que forjan las condiciones entre la esperanza de las oportunidades y las reales entregadas. A su vez, esto se transforma en una coyuntura de investigación a desarrollar en el futuro, integrando modelos de observación de aula en la FID para dar cuenta del nivel de interpretación e implementación de las oportunidades descritas como potenciales en los syllabus de las asignaturas.

\section{CONCLUSIONES}

De acuerdo al trabajo presentado y a los resultados obtenidos, se pueden plantear las siguientes conclusiones principales:

1.- La dimensión de Conocimiento Matemático Escolar de la unidad CARE de aproximación al aula escolar es la única que se observa de forma transversal en los programas de formación inicial de Educación Básica analizados.

2.- No es posible observar una relación coherente entre los indicadores de calidad CARE y los años de acreditación recibida por los programas examinados.

\section{AGRADECIMIENTOS}

Los autores agradecen el programa Fondecyt Postdoctorado №3201094 del Gobierno de Chile.

\section{REFERENCIAS}

American Educational Research Association, American Psychological Association, y National Council on Measurement in Education, Standards for educational and psychological testing, In American Educational Research Association, New Jersey, USA (2014)

Bell, C., y Youngs, P., Substance and show: understanding responses to teacher education programme accreditation processes, https://doi.org/10.1016/j.tate.2010.08.012, Teaching and Teacher Education, 27(2), 298-307 (2011)

Blömeke, S., Hoth, J., y otros 4 autores, Teacher change during induction: development of beginning primary teachers' knowledge, beliefs and performance, https://doi.org/10.1007/s10763-015-9619-4, International Journal of Science and Mathematics Education, 13(2), 287-308 (2015)

Blömeke, S., Kaiser, G., Köning, J., y Jentsch A., Profiles of mathematics teachers' competence and their relation to instructional quality, https://doi.org/10.1007/s11858-020-01128-y, ZDM Mathematics Education 52, $329-342$ (2020)

Cerda, G., Salcedo, P., Pérez, C., y Marín, V., Futuros profesores de matemáticas: rol de la disponibilidad léxica, esquemas de razonamiento formal en logros académicos durante su formación inicial, https://dx.doi.org/10.4067/S071850062017000100005, Formación Universitaria, 10(1), 33-46 (2017)

Comisión Nacional de Acreditación, Criterios de evaluación para la acreditación de carreras profesionales, carreras profesionales con licenciatura y programas de Licenciatura, Santiago, Chile (2017)

Contreras, S., Pensamiento pedagógico en la enseñanza de las ciencias, análisis de las creencias curriculares y sus implicancias para la formación de profesores de enseñanza media, https://dx.doi.org/10.4067/S071850062016000100003, Formación Universitaria, 9(1), 15-24 (2016)

Darling-Hammond, L., Teacher education around the world: what can we learn from international practice?, https://doi.org/10.1080/02619768.2017.1315399, European Journal of Teacher Education, 40(3), 291-309, (2017)

Darling-Hammond, L., Accountability in teacher education, https://doi.org/10.1080/01626620.2019.1704464, Action in Teacher Education, 42(1), 60-71 (2020)

DeGraff, T., Schmidt, C., y Waddell, J., Field-based teacher education in literacy: preparing teachers in real classroom contexts, https://doi.org/10.1080/10476210.2015.1034677, Teaching Education, 26(4), 366-382 (2015)

Deskriptif, K., Panduan, C., y Guru, P., A proposed guide for accreditation of teacher education in developing countries (a descriptive analytical study), Journal Pendidikan Malaysia, 36(1), 11-24 (2012)

Domínguez, M., y Meckes, L., Análisis y propuestas para la acreditación de pedagogías en Chile, https://doi.org/10.4067/S0718-45652011000100009, Calidad en la Educación, 34, 165-183 (2011) 
Grossman, P., Comon, C. y otros 4 autores, Teaching practice: a cross-professional perspective, https://doi.org/10.1177/0022487109336543, Teachers College Record, 111(9), 2055-2100 (2009)

Han S., Blank J., y Berson I., Revisiting reflective practice in an era of teacher education reform: a self-study of an early childhood teacher education program, https://doi.org/10.1080/10901027.2019.1632993, Journal of Early Childhood Teacher Education, 41(2), 162-182 (2020)

Hox, J., Moerbeek, M., y van de Schoot, R., Multilevel analysis: techniques and applications, 3ª Ed., Routledge, New York, USA (2018)

Imig, D., Wiseman, D., Wiseman, A., y Imig, S., What is high quality teacher education?, in Quality and Change in Teacher Education, Professional Learning and Development in Schools and Higher Education by J. Chi-Kin Lee, C. Day, vol. 13, pp. 77-94, Springer, Cham, Alemania (2016)

Ingvarson, L., Beavis, A., y Kleinhenz, E., Factors affecting the impact of teacher education programmes on teacher preparedness: implications for accreditation policy, https://doi.org/10.1080/02619760701664151, European Journal of Teacher Education, 30(4), 351-381 (2007)

Ingvarson, L., y Rowley, G., Quality assurance in teacher education and outcomes: a study of 17 countries, https://doi.org/10.3102/0013189X17711900, Educational Researcher, 46(4), 177-193 (2017)

Kaiser, G., y König, J., Competence measurement in (mathematics) teacher education and beyond: implications for policy, https://doi.org/10.1057/s41307-019-00139-z, Higher Education Policy, 32(4), 597-615 (2019)

Kassambara, A., Practical guide to principal component methods in R: pca, m (ca), famd, mfa, hcpc, factoextra, STHDA (2017)

Klemenz, S., König, J., y Schaper, N., Learning opportunities in teacher education and proficiency levels in general pedagogical knowledge: new insights into the accountability of teacher education programs,

https://doi.org/10.1007/s11092-019-09296-6, Educational Assessment, Evaluation and Accountability, 31(2), 221-249, (2019)

Lee, J., y Day, C., Western and Chinese perspectives on quality and change in teacher education, in Quality and Change in Teacher Education, Professional Learning and Development in Schools and Higher Education by J. Chi-Kin Lee, C. Day, vol. 13, 1-16, Springer, Cham, Alemania (2016)

Ling, L., Australian teacher education: inside-out, outside-in, backwards and forwards?, https://doi.org/10.1080/02619768.2017.1385599, European Journal of Teacher Education, 40(5), 561-571 (2017)

Lopez, C., Benedito, V. y León M., Enfoque de competencias en la formación universitaria y su impacto en la evaluación, La perspectiva de un grupo de profesionales expertos en pedagogía, https://dx.doi.org/10.4067/S071850062016000400003, Formación Universitaria, 9(4), 11-22 (2016)

Mayer, D., Dixon, M., y otros 6 autores, Studying the effectiveness of teacher education: early career teachers in diverse settings, Springer, Singapore (2017)

Mayring, P., Qualitative content analysis: theoretical background and procedures, in approaches to qualitative research in mathematics education, Advances in Mathematics Education by A. Bikner-Ahsbahs, C. Knipping, N. Presmeg, 365380, Springer, Dordrecht (2015)

Placier, P., Letseka, M. y otros 5 autores, The history of initial teacher preparation in international contexts, in International handbook of teacher education by J. Loughran, M. Hamilton, pp. 23-68, Springer, Singapore (2016)

Pushpanadham, K. (Ed.), Teacher education in the global era: perspectives and practices, Springer Nature, Singapore, (2020)

Romanowski, M., y Alkhateeb, H., The mcDonaldization of CAEP accreditation and teacher education programs abroad, https://doi.org/10.1016/j.tate.2020.103028, Teaching and Teacher Education, 90, 1-7, (2020)

Rowe, E., y Skourdoumbis, A., Calling for 'urgent national action to improve the quality of initial teacher education': the reification of evidence and accountability in reform agendas, https://doi.org/10.1080/02680939.2017.1410577, Journal of Education Policy, 34(1), 44-60 (2017)

Solbrekke, T., y Sugrue, C., Professional accreditation of initial teacher education programmes: teacher educators' strategies between "accountability" and "professional responsibility"?, https://doi.org/10.1016/j.tate.2013.07.015, Teaching and Teacher Education, 37, 11-20 (2014)

Talbot, D., Evidence for no-one: standards, accreditation, and transformed teaching work, https://doi.org/10.1016/j.tate.2016.05.006, Teaching and Teacher Education, 58, 80-89 (2016)

Werler, T., y Tahirsylaj, A., Differences in teacher education programmes and their outcomes across Didaktik and curriculum traditions, https://doi.org/ 10.1080/02619768.2020.1827388, European Journal of Teacher Education, 43, 119, (2020) 
